\title{
AUTOMATED FISH CAGES INVENTORYNG AND MONITORING USING H/A/ $\alpha$ UNSUPERVISED WISHART CLASSIFICATION IN SENTINEL 1 DUAL POLARIZATION DATA
}

\author{
Ariel Russell G. ${ }^{1}$, Denisse Castillo U. ${ }^{1}$, Sebastián Elgueta A. ${ }^{1}$, Carlos Sierralta J. ${ }^{1}$ \\ ${ }^{1}$ Superintendencia del Medio Ambiente del Gobierno de Chile
}

KEY WORDS: Fish cages monitoring, Aquaculture monitoring, Sentinel 1, PolSAR, Dual-Pol, H/A/ $\alpha$ decomposition, Unsupervised Wishart Classifier.

\begin{abstract}
:
Aquaculture fish cages are usually located remote areas with poor accessibility. Synthetic Aperture Radar (SAR) is relevant to register and monitor their activity. However, in order to take advantage of the large amount of information this technology produce, it is necessary to develop automated tools of analysis. This work proposes an automated approach through an unsupervised polarimetric classification method using Sentinel 1 IW SLC Dual-Pol (VV + VH) products. An experimental evaluation was applied in Calbuco, an area with intense aquaculture activity in southern Chile. It was possible to demonstrate that this approach allows to improve the capacity of classification of previous experiences ( $86.49 \%$ user's accuracy and $96.97 \%$ producer's accuracy). Further studies are required to know the impact of wind speed on the classification as well as the spatial precision of the detection.
\end{abstract}

\section{INTRODUCTION}

Aquaculture facilities using fish cage are typically located in areas of difficult access such as fjords and straits. Because of the above, remote sensing can be used as a useful technology to detect and monitor these structures. There are several experiences using satellite remote sensing to detect aquaculture fish cages with passive sensors (Zhang et al., 2010; Peralta et al., 2015) and active Synthetic Aperture Radar (SAR) systems (Steckler, 2001; Travaglia, et al., 2004; Sierralta et al., 2015). The SAR experiences are remarkable due its measurement capacity in different atmospheric conditions; nevertheless, these studies only use backscattering amplitude discarding phase, disregarding relevant information to improve detection. Only Stecker (2003) propose a supervised classification (88\% producer's accuracy) instead Travaglia et al. (2004) and Sierralta et al. (2015) uses SAR only for photointerpretation.

Based on this review, the main advantage of detecting fish cages facilities with SAR is the difference in the backscattering amplitude of the cages respect to the marine surface that surrounds them. This is explained by the backscattering mechanism for each surface, where the sea surface, under calm conditions tends to present a specular scattering versus other mechanisms. In this direction, Polarimetric SAR is a useful tool to classify backscattering mechanisms, using complex SAR data, considering amplitude and phase (Lee \& Pottier, 2009).

At the same time, advances in access to Earth Observation (henceforth EO), as well as the capacity to deliver and process information have increased significantly (Durrieu \& Nelson, 2013), pushing new tools for automated big data analysis (Marchetti et al., 2016; Casu et al., 2017). This, together with the increasing availability of EO Open Data programs (Harris \& Baumann, 2015) allow us to project programs for continuous monitoring in several fields. The SAR Sentinel $1 \mathrm{~A}$ and B platforms of the Copernicus Program are an example of this trend.

Sentinel 1 platforms are monostatic SAR C-band systems that have a continuous and regular acquisition mode called Interferometric Wide Swath (IW), which is applied across most of the world territory and has a dual polarimetric configuration
$(\mathrm{VV}+\mathrm{VH})$. Typically, the description of the scattering mechanisms require full polarimetric information (VV+VH+HH+HV) (Lee \& Pottier, 2009; Ji \& Wu, 2015), however, in recent works several techniques have been studied to extract the scattering mechanisms from Dual-Pol information (Cloude, 2007; Ainsworth et al., 2009; Cloude et al., 2012, Raney, 2016; Zakeri, 2017). One of the most frequently used mechanisms to extract the scattering mechanisms is Polarimetric Decomposition (Cloude \& Pottier, 1997) based in covariance matrix (C) applying Unsupervised Wishart Classification (Lee et al., 1999a) over the original Polarimetric Decomposition. This generates an iterative clustering process considering the $\mathrm{C}$ matrix present a Wishart distribution, which allows a probabilistic clustering function (Lee \& Grunes, 1994; Lee et al., 1994).

This paper presents an evaluation of a procedure to classify fish cages into Sentinel 1 IW Single Look Complex (SLC) Dual-Pol products, verifying if these facilities are possible to extract by the scattering mechanisms (adding some simplified GIS processes). Scattering mechanisms are classified by an unsupervised classification of wishart of matrix $\mathrm{C}$, using a polarimetric decomposition H/A/ $\alpha$ (Entropy, Anisotropy and Alpha mean angle). The evaluation was applied over Calbuco area in southern Chile, a region with a big aquaculture fish activity of Salmon. Finally, it is discussed how this experience helps to expand the state of the art in this topic and challenges for future research are highlighted.

\section{STUDY AREA AND DATA}

\subsection{Study area}

The study area corresponds to the sector of Calbuco, an area of intense production of salmonids (in fish cages) and other types of aquaculture activities in Los Lagos Region, Chile. 

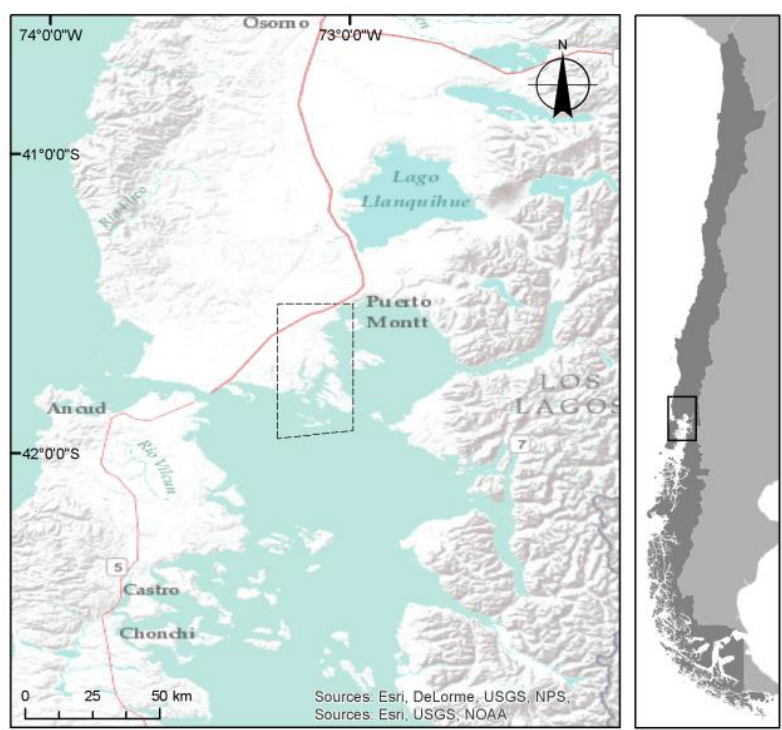

Figure 1. Study area.

\subsection{SAR Sentinel 1 data}

For the analysis, two Sentinel 1 IW SLC Dual-Pol $(\mathrm{VV}+\mathrm{VH})$ SAR descending images were used from S1B platform, acquired in April 4, 2017 and April 16, 2017², 9 and 3 days of difference with validation data, respectively.

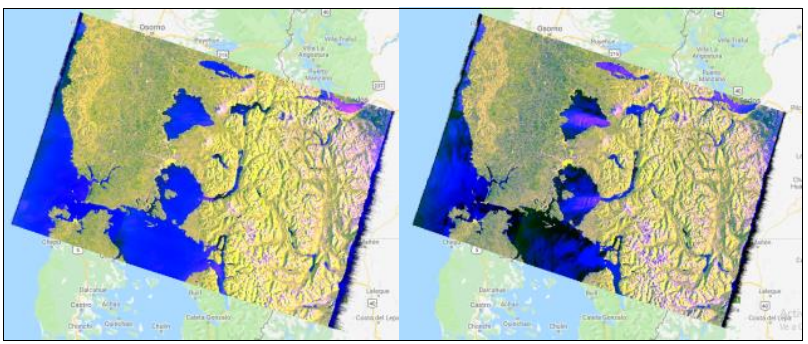

Figure 2. Composite preview of SAR S1B used. In left April 4, 2017, image. In Right April 16, 2017, image.

\subsection{Accuracy assessment data}

A cloud free RGB composite of a very high-resolution image available for open visualization in Google Earth Pro platform was used to estimate the accuracy of classified object. The image was acquired by WorldView-2 satellite in April 13, 2017 with $50 \mathrm{~cm}$ of with spatial resolution.

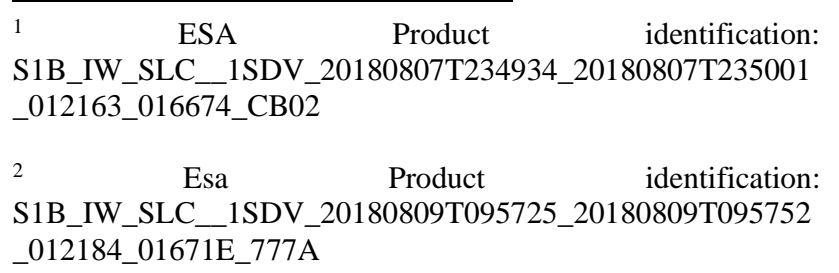

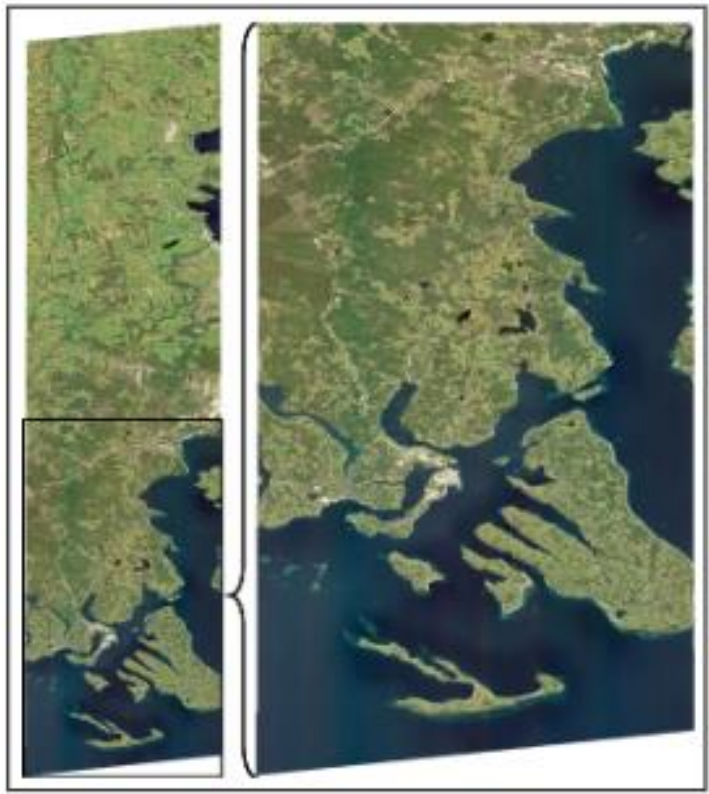

Figure 3. RGB composite of WorldView-2 image ID_103001006851BD00.

\subsection{Software tools}

For the pre-processing of the Scattering matrix of Sentinel 1 IW SLC products and the final geocoding process, we use the "Sentinel 1 Toolbox" (S1TBX) V6.0 (Array System Computing, 2019). For the covariance matrix computing, speckle filtering, polarimetric decomposition and unsupervised Wishart Classification, we use the "Polarietric SAR Data Processing and Educational Tool" (PolSARPro) V5.1.3 developed by Institute of Electronics and Telecommunications of Rennes (IETR, 2019).

\section{METHODS}

The proposed method is divided into 3 main stages; I) Sentinel 1 IW SLC pre-processing to get a clean Scattering matrix (S) available for polarimetric processes; II) Polarimetric processes from the matrix $\mathrm{S}$, at this stage the speckle reduction and dispersion mechanism extraction is performed; III) postprocessing, including the geocoding of the results and a flow of GIS processes to create a layer of fish cages based on extent of polygons. Finally, a confusion matrix is applied to determine the accuracy of classification process.

\subsection{Pre-processing SLC Sentinel 1 IW data}

Chain of procedures for obtaining the S-matrix, some processes are specific for SAR S1 IW SLC products, due to the particularities of the acquisition mode "Terrain Observation with Progressive Scans SAR" (TOPSAR) (De Zan \& Guarnieri, 2006).

3.1.1 TOPSAR-Split: Each S1 IW SLC product is composed of three sub-swaths. This process allows you to select and separate the sub-swath with which is wanted to work with.

3.1.2 Orbit-file metadata restitution: This process updates the orbit metadata of each product by assigning measured orbit data. 
3.1.3 Radiometric calibration: This process transforms the complex values (real and imaginary components) of the observation to physical values of the received signal.

3.1.4 TOPSAR-deburst: Bursts are a residual phenomenon of the IW acquisition mode present in S1 IW SLC products, TOPSAR-Deburst eliminates this effect.

Finally, a physical Scattering Matrix (1) is computed for a dual polarization SAR data, assuming the reciprocity between HV and HV signals (Ji \& Wu, 2015).

$$
S=\left[\begin{array}{cc}
0 & S_{H V} \\
S_{V H} & S_{V V}
\end{array}\right]
$$

\subsection{Scattering mechanism cluster from Dual-Pol VV+VH scattering matrix}

3.2.1 Covariance Matrix $\left(C_{2}\right)$ is a specific case of matrix $C_{3}$ (Quad-pol), for its calculation it is necessary to define the dispersion vector $\Omega$ present in (2) (Ji \& Wu, 2015).

$$
\Omega=\left[S_{V V}, 2 S_{V H}\right]^{T}
$$

where $T=$ Transpose matrix operator.

Nielsen et al. (2017) defines $\mathrm{C}_{2} \mathrm{VV}+\mathrm{VH}$ matrix as (3).

$$
C_{2}=\Omega * \Omega^{H}=\left[\begin{array}{ll}
\left\langle S_{V V} S_{V V}^{*}\right\rangle & \left\langle S_{V V} S_{V H}^{*}\right\rangle \\
\left\langle S_{V H} S_{V V}^{*}\right\rangle & \left\langle S_{V H} S_{V H}^{*}\right\rangle
\end{array}\right]
$$

where

$$
\begin{aligned}
& H=\text { Conjugated transpose operator. } \\
& \langle>\quad=\text { Ensemble average operator. } \\
& * \quad=\text { Complex conjugate operator. }
\end{aligned}
$$

3.2.2 Polarimetric speckle filtering: Speckle is a phenomenon typical of SAR products, due to the incoherence in phase in rough surfaces at the wavelength scale (Lee \& Pottier, 2009). This phenomenon also affects the accuracy of polarimetric classification processes (Lee et al., 1999b). In the case of the detection of fish cages, this process is relevant, since the roughness of the marine surface is subject to the formation of waves of capillarity, product of the wind (Harding et al., 2001; Vachon et al., 2004).

There are several polarimetric speckle filters and each one has specific advantages. The comparative analysis of Farage et al. (2007), Turkar (2010), Boutarfa et al. (2015), Mousavi et al. (2005) and Medasani \& Reddy (2017) was reviewed. The Lee Refined Speckle Filter (Lee et al., 2006) was chosen, because in the comparative analyzes it proved to be useful for edge preservation (Mousavi et al., 2015; Medasani \& Reddy, 2017) and to improve the accuracy of polarimetric classifications (Farage et al., 2007; Turkar, 2009). Specifically, it was decided to apply this filter with a $7 \times 7$ mobile window.

3.2.3 H/A/a Dual Polarimetric decomposition: Scattering mechanisms classification based on polarimetric decomposition theorems in Dual-Pol SAR products are not recommended (Lee \& Pottier, 2009; Ji \& Wu, 2015), however, they are useful for probabilistic clustering functions such as unsupervised wishart classification. Entropy (H), Anisotropy (A) and Scattering mean angle $(\alpha)$ parameters are calculated from the decomposition of eigenvalues and eigenvectors of $\mathrm{C}_{2}$ matrix in (4) (Pelich et al., 2018).

$$
C_{2}=\left[U_{2}\right]\left[\begin{array}{ll}
\lambda_{1} & 0 \\
0 & \lambda_{2}
\end{array}\right]\left[U_{2}\right]^{* T}
$$

where $\quad \lambda_{1}, \lambda_{2}=$ Eigenvalues of covariance matrix.

$U_{2}=$ Eigenvector of covariance matrix

Entropy $(\mathrm{H})$ represents the degree of statistical disorder of each scattering mechanism, when its value is close to 1 it is more uncertain the dominant backscattering mechanism, can be calculated with (5) (Lee \& Pottier, 2009).

$$
\begin{gathered}
H=-\sum_{i=1}^{2} P_{i} \log _{2}\left(P_{i}\right) \\
P_{i}=\frac{\lambda_{i}}{\lambda_{1}+\lambda_{2}}
\end{gathered}
$$

where $P_{\mathrm{i}}=$ The pseudoprobabilities, representing the importance of eigenvalues with respect to the scattered power (Pelich et al., 2018).

Anisotropy (A) is complementary to $\mathrm{H}$ and describes the relative importance of secondary or tertiary eigenvalues (Ouarzeddine et al., 2007), has been shown to significantly improve the quality of unsupervised polarimetric classification processes and is represented in (7) (Lee \& Pottier, 2009).

$$
A=\frac{\lambda_{1}-\lambda_{2}}{\lambda_{1}+\lambda_{2}}
$$

Mean scattering angle $(\alpha)$ explain the type of scattering, where low values (close to 0 ) are associated with specular dispersion, while high values (close to 90) explain double-bounce scattering. It can be calculated with (8) (Pelich et al., 2018).

$$
\alpha=\sum_{i=1}^{2} P_{i} \alpha_{i}
$$

3.2.3 Unsupervised H/A/a Wishart Classification: This solution, proposed by Lee et al. (1999a) improves the classification, due the matrix $\mathrm{C}$ conforms to Wishart distribution thus the scattering mechanisms can be grouped by Maximum Likelihood Estimation (MLE), based on Wishart distribution. The product of performing this procedure in the $\mathrm{H} / \mathrm{A} / \alpha$ space generates 16 classes. For this procedure, iterations were limited to a maximum of 5 or until less than $5 \%$ of the pixels change from one class to another for each iteration, due hardware limitation criteria.

\subsection{Post-processing}

The scattering mechanisms grouped into 16 classes are in slantrange system, in which the geocoding procedure for EnvisatASAR are projected to ground (Small \& Schubert, 2008) with values interpolated by nearest neighbors. 
In a GIS environment, a low tide water mask is applied to conserve only the marine surface. The scattering clusters whose presence is less than $0.8 \%$ (experimental value for the study area) are reclassified, so the classes will correspond to backscattering types of floating structures differs from the sea surface. Then raster is vectorized and the area and semi-major axis of polygons are calculated. Those polygons whose dimensions are similar to the dimensions of the fish cages are selected (3,600-30,000 $\mathrm{m}^{2}$ and 90-370 $\mathrm{m}$ length).

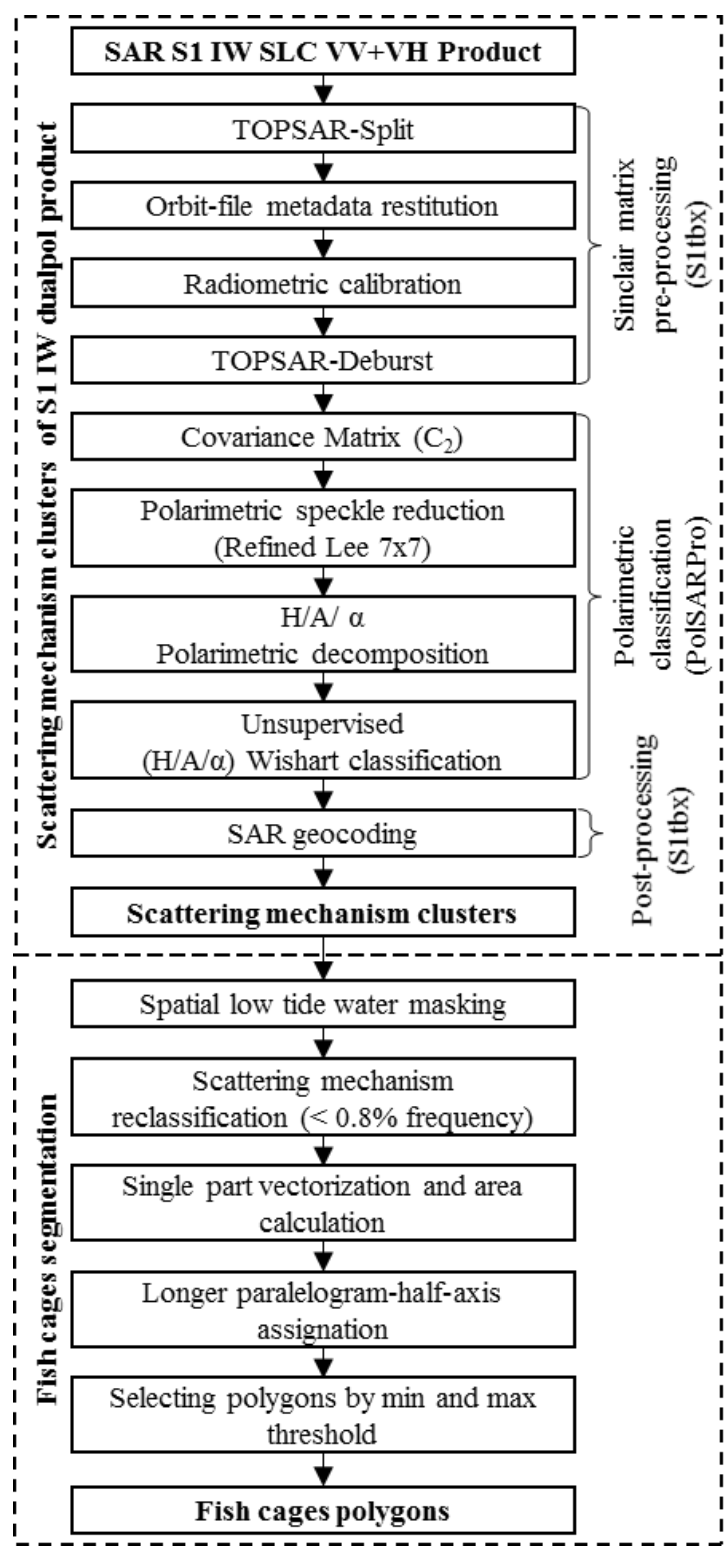

Figure 4. Proposed processing flowchart.

\section{RESULTS AND DISCUSSION}

\subsection{Scattering clusters overview}

In Figure 5 it can be seen that the scattering mechanism clustering after the unsupervised classification and masking, shows the presence of structures associated with aquaculture production, including fish cages and of other types of aquaculture structures, differentiating from marine surface.

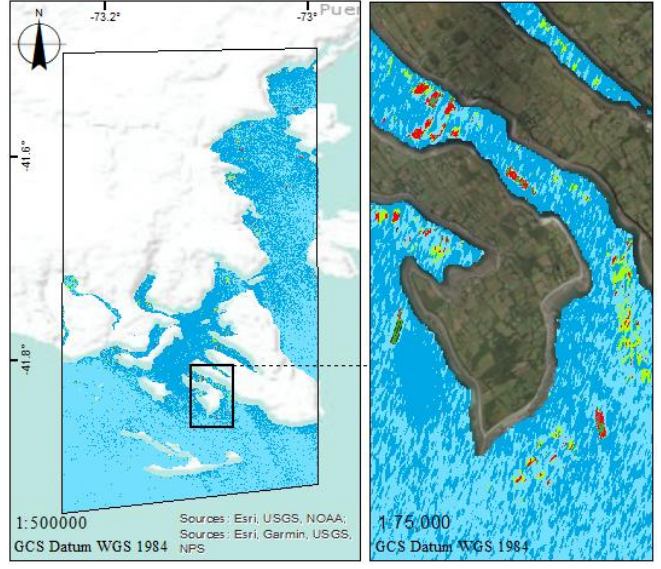

Figure 5. Masked scattering clusters.

\subsection{Fish cages scattering group and vectorizing}

Observing the process of vectorization, it is possible to point that the fish cages were detected integrally, as well as other smaller floating structures such as accessory aquaculture floating objects.
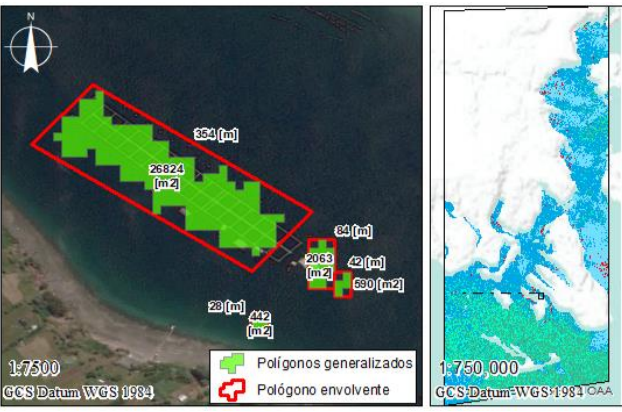

Figure 6. Vectorized scattering groups.

\subsection{Classification accuracy}

The validation data used contains 33 fish cages and a nonspecific number of other floating structures of various dimensions and materials. In both SAR products, 32 fish cages were detected. In the case of false positives, the product of April 4, 2017, 7 misclassified objects were detected, while on April 16,2017 , only three misclassified objects were detected. In both products, the producer accuracy of the Stecker (2003) experience was higher.

\begin{tabular}{cccc} 
Accuracy & Abril 4 & Abril 16 & Both SAR \\
measurement & 2017 & 2017 & products \\
\hline User's accuracy & $82,05 \%$ & $91,43 \%$ & $86,49 \%$ \\
$\begin{array}{c}\text { Produces } \\
\text { accuracy }\end{array}$ & $96,97 \%$ & $96,97 \%$ & $96,97 \%$
\end{tabular}

Table 1. Confusion matrix accuracy measurements.

\subsection{PolSAR approach versus previous experiences}

In Figure 7 it can be seen that the use of PolSAR classification techniques improves the detection capacity of fish cages, in comparison to the amplitude use only (SAR S1 calibrated and Refined Lee speckle filtered). 

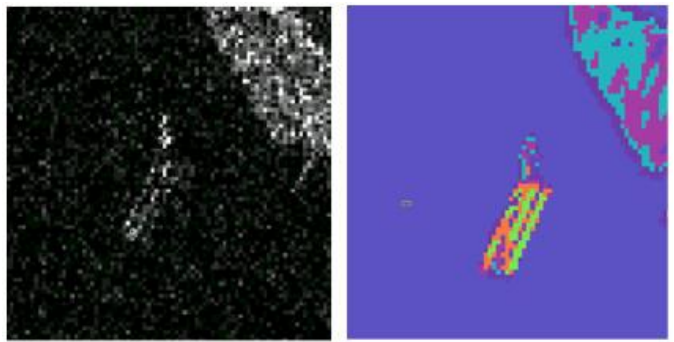

Figure 7. Amplitud backscattering approach (left) and polarimetric classification (right).

\subsection{Unclassified fish cages}

In both SAR products, one fish cage was not detected. Reviewing the validation image, it was found that this fish cage had a round structure and whose material is different from the rest of the facilities detected. In Figure 8 it can be seen that only some pixels were associated with scattering mechanisms of aquaculture structures and geometric nature of bounce, so that most of these were classified as marine surface.
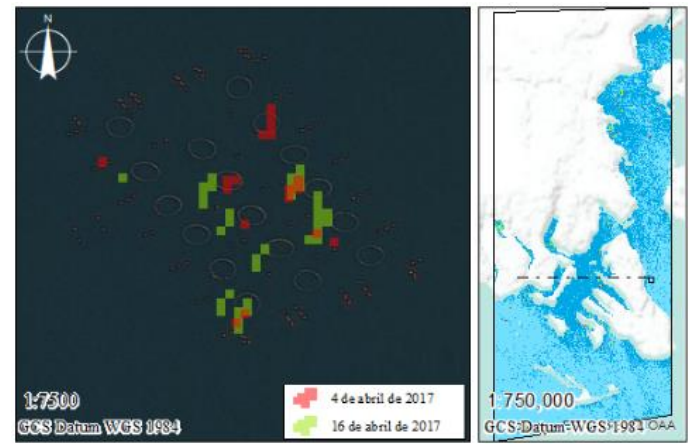

Figure 8. Undetected fish cages.

\subsection{Additional considerations}

The differences in the user's accuracy for the two SAR products analyzed suggest that there are differences in the ground conditions that modify the performance of the procedure. One of the possible causes is the wind on the sea surface, since this can change the roughness conditions at the wavelength scale. It would be relevant to know the wind velocity at the time of acquisition of the SAR product in order to deepen on the impact of this variable.

Another aspect to consider is spatial precision, this aspect is not considered in the study due to the need of positioning data at the time of SAR acquisition, so the spatial precision will have a referential character until it is considered that aspect in future works.

\section{CONCLUSIONS}

The use of PolSAR techniques allows better performance than the use of the backscattering coefficient to detect fish cages. The limitations of Dual-Pol products to extract the dispersion mechanisms in a polarimetric decomposition can be mitigated with procedures such as $\mathrm{H} / \mathrm{A} / \alpha$ Unsupervised Wishart Classification, allowing the use of these techniques to be extended operationally in products such as of the Sentinel 1 IW
SLC. It is necessary to evaluate in greater depth the impact of variables such as wind speed in the processes of extraction of scattering mechanisms in the sea. To estimate the spatial precision of this procedure it is necessary to have field data in future works.

\section{REFERENCES}

Ainsworth, T. L., Kelly, J. P., Lee, J.-S., 2009. Classification coparison between dual-pol, compact polarimetric and quad-pol SAR imagery. ISPRS Journal of Photogrammetry and Remote Sensing, 64(5), 464-471.

Array System Computing, 2019. Sentinel 1 Toolbox. Version 6.0. European Space Agency (ESA). https://sentinel.esa.int/web/sentinel/toolboxes/sentinel-1 (27 October 2019).

Boutarfa, S., Bouchemakh, L., Smara, Y., 2015. Comparative Study of Speckle Filtering Methods in PolSAR Radar Images. PolInSAR 2015, Proceedings of the conference. ESA SP-729, id.65, 1-6. ISBN: 978-92-9221-293-3.

Casu, F., Manunta, M., Agram, P. S., Crippen, R. E., 2017. Big Remotely Sensed Data: Tools, applications and experiences. Remote Sensing of Environment, 202, 1-2.

Cloude, S. R., Pottier, E., 1997: An entropy based classification scheme for land applications of polarimetric SAR. IEEE Transactions on Geoscience and Remote Sensing, 35(1), 68-78.

Cloude, S. R., 2007. The dual polarization entropy/alpha decomposition: A PalSAR case Study. PolInSAR 2007 Proceedings of the conference. 1-6. https://earth.esa.int/workshops/polinsar2007/papers/75_cloude.p df.

Cloude, S. R., Goodenough, D. G., Chen, H., 2012: Compact Decomposition Theory. IEEE Geoscience and Remote Sensing Letters, 9(1), 28-32.

De Zan, F., Guarnieri, A. M., 2006: TOPSAR: Terrain Observation by Progressive Scans. IEEE Transactions on Geoscience and Remote Sensing, 44(9), 2352-2360.

Durrieu, S., Nelson, R., 2013: Earth observation from space: The issue of environmental sustainability. Space Policy, 29(4), 238-250.

Farage, G., Foucher, S., Benie, G., 2007: Comparison of PolSAR Speckle Filtering Techniques. IGARSS 2006 (IEEE Conference Proceedings). 1760-1763. doi.org/10.1109/IGARSS.2006.455.

Harding, L. W., Miller, W. D., Swift, R. N., Wright, C. W., 2001. Aircraft Remote Sensing. Encyclopedia of Ocean. 113122. Academic Press. Woods Hole, Massachusetts, USA.

Harris, R., Baumann, I., 2015. Open data policies and satellite Earth observation. Space Policy, 32, 44-53.

IETR. 2019. Polarimetric SAR data Processing and Education Toolbox. University of Rennes. https://www.ietr.fr/polsarprobio/ (27 October 2019). 
Ji, K., Wu, Y., 2015. Scattering Mechanism Extraction by a Modified Cloude-Pottier Decomposition for Dual Polarization SAR. Remote Sensing, 7(6), 7447-7470.

Lee, J. S., Grunes, M. R., 1994. Classification of multi-look polarimetric SAR data based on complex Wishart distribution. NTC-92: National Telesystems Conference [Proceedings]. 7/21-7/24. doi.org/10.1109/NTC.1992.267879.

Lee, J.-S., Hoppel, K. W., Mango, S. A., Miller, A. R., 1994. Intensity and phase statistics of multilook polarimetric and interferometric SAR imagery. IEEE Transactions on Geoscience and Remote Sensing, 32(5), 1017-1028.

Lee, J.-S., Grunes, M. R., Ainsworth, T. L., Du, L.-J., Schuler, D. L., 1999a. Unsupervised Classification Using Polarimetric Decomposition and the Complex Wishart Classifier. IEEE Transactions on Geoscience and Remote Sensing, 37(5), 2249. 2258.

Lee, J.-S., Grunes, M. R., Grandi, G., 1999b. Polarimetric SAR speckle filtering and its implication for classification. IEEE Transactions on Geoscience and Remote Sensing, 37(5), 23632373.

Lee, J.-S., Grunes, M. R., Schuler, D.L., Pottier, E., FerroFamil, L., 2006. Scattering-model-based speckle filtering of Polarimetric SAR data. IEEE Transactions on Geoscience and Remote Sensing, 44(1), 176-187.

Lee, J.-S., Pottier, E., 2009. Polarimetric Radar Imaging: From Basics to Applications. CRC Press, Florida, USA.

Medasani, S., Reddy, G. U., 2017. Analysis and Evaluation of Speckle Filters for Polarimetric Synthetic Aperture (PolSAR) Data. International Journal of Applied Engineering Research, 12(15), 4916-4927.

Mousavi, M., Amini, J., Maghsoudi, Y., Arab, S., 2015. PolSAR Speckle Filtering Techniques and Their Effects on Classification. IGTF 215 - ASPRS Annual Conference. 91-96. Nielsen, A. A., Conradsen, K., Skriver, H., Canty, M. J., 2017. Change detection in a series of Sentinel-1 SAR data. 2017 9th International Workshop on the Analysis of Multitemporal Remote Sensing Images (MultiTemp), 1-3. doi.org/10.1109/Multi-Temp.2017.8035210.

Nielsen, A. A., Conradsen, K., Skriver, H., Canty, M. J., 2017. Change detection in a series of Sentinel-1 SAR data. MultiTemp 2017. 1-3. doi.org/10.1109/Multi-Temp.2017.8035210.

Ouarzeddine, M., Souissi, B., Belhadj-Aissa, A., 2007. Unsupervised Classification Using Wishart Classifier. Proceedings PolInSAR 2007. ISBN: 92-9291-208-7.

Pelich, R., Lopez-Martinez, C., Chini, M., Hostache, R., Matgen, P., Ries, P., Eiden, G., 2018. Exploring DualPolarimetic Descriptors for Sentinel-L Based Ship Detection. $\begin{array}{lll}\text { IGARSS 2018, 2404-2407. } & \end{array}$ doi.org/10.1109/IGARSS.2018.8517906.

Peralta, R. V., Jalbuena, R. L., Curz, C. A., Tamondong, A. M., 2015. Development of a semi-automated object-based classification technique for extraction of aquaculture features using LiDAR and WorldView-2 satellite image data. ACRS 2015, 2195-2201. doi.org/10.1109/r10-htc.2016.7906855.
Raney, R. K., 2016. Comparing Compact and Quadrature Polarimetric SAR Performance. IEEE Geoscience and Remote Sensing Letters, 13(6), 861-864.

Sierralta, C., Garay, C., Ramírez, H., Sepúlveda, G., 2015. Enforcing aquaculture in southern Chile through SAR imagery. Special Report on Next Generation Compliance, International Network for Environmental Compliance and Enforcement/Institute for Governance \& Sustainable Development, Washington, DC, USA.

Small D., Schubert, A., 2008. Guide to ASAR Geocoding. Tech. Rep. RSL-ASAR-GC-AD 1.01, Remote Sensing Laboratories, University of Zurich, Zurich, Switzerland.

Steckler, C. D., 2003. Using Radarsat to Detect and Monitor Stationary Fishing Gear and Aquaculture Gear on the Eastern Gulf of Thailand. Department of Geography, University of Victoria. Victoria, Canada.

Travaglia, C., Giuliana, G., Aguilar-Manjarrez, J., Lopez, N. A., 2004. Mapping coastal aquaculture and fisheries structures by satellite imaging radar: Case study of the Lingayen Gulf, the Philippines. FAO Fisheries Tech. Paper $\mathrm{N}^{\circ} 459$, Publishing Management Service, FAO. Rome, Italy.

Turkar, V., 2010. The effects of different filters on classification accuracy of polarimetric SAR data acquired from SIR-C, and ALOS PALSAR. ICAdC-2009. Ch 2, 73-77.

Vachon, P. W., Monaldo, F. M., Holt, B., Lehner, S., 2004. Ocean Surface Waves and Spectra. Synthetic Aperture Radar Marine User's Manual. Office of Research and Applications, NOAA. Ch 5, 139-170. Washington, DC, USA.

Zakeri, H., 2017. Application of Polarimetric Synthetic Aperture Radar Imagery for Land Cover Classification of Urban and Suburban Areas. Chiba University. 115p. Chiba, Japan.

Zhang, T., Li, Q., Yang, X., Zhou, C., Su, F., 2010. Automatic mapping aquaculture in coastal zone from TM imagery with OBIA approach. 2010 18th International Conference on Geoinformatics. $1-4$. doi.org/10.1109/GEOINFORMATICS.2010.5567961. 